Received 1 December 1980; accepted 9 January 1981.

1. Jordan, C., Brueckner, G. E., Bartoe, J-D. F., Sandlin, G. D. \& VanHoosier, M. E. Nature 270, 326-327 (1977).

2. Beckwith, S., Gatley, I., Matthews, K. \& Neugebauer, G. Astrophys. J. Lett. 223, L 41-L43 (1978).

3. Kuhi, L. V. Astrophys. J. 140, 1409-1433 (1964).

4. Schwartz, R. D. Astrophys. J. 191, 419-432 (1974).

5. Brown, A. \& Jordan, C. Mon. Not. R. astr. Soc. (in the press)

6. Gondhalekar, P. M., Penston, M. V. \& Wilson, R. Proc. Symp. The First Year of IUE (ed. Willis, A. J.) 109-116 (University College London, 1979).

7. Gahm, G. F., Fredga, K., Liseau, R. \& Dravins, D. Astr. Astrophys. 73, L4-L6 (1979).

8. Imhoff, C. L. \& Giampapa, M. S. Astrophys. J. Lett. 239, L 115-L119 (1980).

9. Herzberg, G. \& Howe, L. L. Can. J. Phys. 37, 636-659 (1959)

10. Allison, A. C. \& Dalgarno, A. Alomic Data 1, 289-304 (1970).

11. Ashwartz, R. D. Astrophys. J. 195, 631-642 (1975)

12. Maran, S. P., Hobbs, R. W., Brown, R. L., Jura, M. \& Knapp, G. R. Astr. J. 84, 1709-1712 (1979).

13. Hollenbach, D. J. \& Shull, J. M. Astrophys. J. 216, 419-426 (1977).

14. London, R., McCray, R. \& Chu, S. I. Astrophys. J. 217, 442-447 (1977).

15. Kwan, J. Astrophys. J. 216, 713-723 (1977).

16. Osterbrock, D. E. Astrophys. J. 136, 359-364 (1962)

17. Black, J. H. \& Dalgarno, A. Astrophys. J. 203, 132-142 (1976).

17. Black, J. H. \& Dalgarno, A. Astrophys. J. 203,
18. Shull, J. M. Astrophys. J. 219, 877-885 (1978).

18. Shull, J. M. Astrophys. J. 219, 877-885 (1978).

Jordan, C., Brueckner, G. E., Bartoe, J-D. F., Sandlin, G. D. \& VanHoosier, M. E. Astrophys. J. 226, 687-697 (1978).

20. Shull, J. M. Astrophys. J. 224, 841-847 (1978).

21. Herbig, G. H. Astrophys. J. 214, 747-758 (1977).

22. Turner, J., Kirby-Docken, K. \& Dalgarno, A. Astrophys. J. Suppl. 35, 281-292 (1977).

23. Feldman, P. D. \& Fastie, W. G. Astrophys. J. Lett. 185, L101-L104 (1973).

24. Hollenbach, D. J. \& McKee, C. F. Astrophys. J. Lett. 241, L47-L50 (1980)

25. McClintock, W., Henry, R. C., Moos, H. W. \& Linsky, J. L. Astrophys. J. 202, 733-740 (1975).

\section{A water vapour maser in the Large Magellanic Cloud}

\section{E. Scalise Jr}

CRAAM/INPE: Instituto de Pesquisas Espaciais, Conselho Nacional de Desenvolvimento, Cientifico e Tecnológico-CNPq, CP 515, 12200-São José dos Campos, SP, Brasil

\section{A. Braz}

CNPq-Observatório Nacional, Rua Pará, 277, 01243-São Paulo, SP, Brasil

Surveys of the Large Magellanic Cloud (LMC), the closest satellite galaxy to our Solar System, for water vapour sources of measurable intensity have so far yielded negative results. These searches, by Johnston et $a l^{1}{ }^{1}$ and Kaufmann et $a l^{2}$, with detection limits of about 150 and $20 \mathrm{Jy}\left(1 \mathrm{Jy}=10^{-26} \mathrm{Wm}^{-2} \mathrm{~Hz}^{-1}\right)$, respectively, suggest that any water vapour masers that exist in the LMC are much weaker than the sources recognized in our own Galaxy and in several more distant galaxies ${ }^{3-5}$. We now report the results of a survey using the 45 -ft Itapetinga radio telescope which shows the presence of a water vapour maser in the N159 region of the LMC.

The survey was carried out in 1979-80. Upper detection limits of about $1.2 \mathrm{Jy}$ were attained with the use of long integration times and a maser amplifier front-end, the total system temperature ranging from 250 to $400 \mathrm{~K}$. Spectral information was provided by a 47-channel spectrometer, $100-\mathrm{kHz}$ bandwidth filter bank, giving a velocity resolution of $1.35 \mathrm{~km}$ $\mathrm{s}^{-1}$. Two vertically polarized feed horns were used in beamswitching the on-off observing mode. Each independent observation was taken with $30 \mathrm{~min}$ integration. We selected observations made in good weather conditions and our data were corrected for atmospheric attenuation effects by assuming an average atmospheric optical depth of 0.2 . The data were also corrected for random attenuation and elevation gain dependence.

We concentrated our search for the $J=6_{16}-5_{23}$ transition of the water maser line $(f=22235.08 \mathrm{MHz})$ in two of the strongest $\mathrm{H}$ (II) regions in the $\mathrm{LMC}^{6}-\mathrm{N} 157$ (ref. 7,30 Doradus) and N159 - and in two dark nebulae ${ }^{8}$-Hodge 47 and Hodge 52. Huggins et al..$^{9}$ studied these four regions in their carbon monoxide survey.
Water vapour emission was detected in only one of these four positions, N159, and its spectrum is shown in Fig. 1. It presents two components, one $8 \mathrm{~km} \mathrm{~s}^{-1}$ wide with a narrow feature of $7.2 \mathrm{Jy}$ at $233.6 \mathrm{~km} \mathrm{~s}^{-1}$, and the other with a feature of $3.2 \mathrm{Jy}$ at $213.9 \mathrm{~km} \mathrm{~s}^{-1}$.

It is interesting that the water maser we discovered in N159 coincides with the lowest colour temperature far-IR source detected by Werner et al. ${ }^{10}$ in the LMC-30 Doradus-which is also the strongest source. A large $\mathrm{CO}$ cloud, $24 \mathrm{pc}$ in diameter, was found in this direction'. Other molecules such as $\mathrm{H}_{2} \mathrm{CO}$ (ref. 11), $\mathrm{OH}$ (ref. 12) and $\mathrm{CO}$ (ref. 9), have been discovered at this position in the LMC, with velocities almost coincidental with the $\mathrm{H}_{2} \mathrm{O}$ velocity.

Regarding the intensity of this maser, it would be a strong source of $8 \times 10^{4} \mathrm{Jy}$ if placed at the distance of the Orion Nebula, but when compared with the W49 masers, it is much weaker in terms of total luminosity ${ }^{13}$.

A negative result was obtained for the 30 Doradus nebula, probably due to the fact that its core contains a large number of young $\mathrm{OB}$ stars ${ }^{14}$ responsible for the ionization of the molecules.

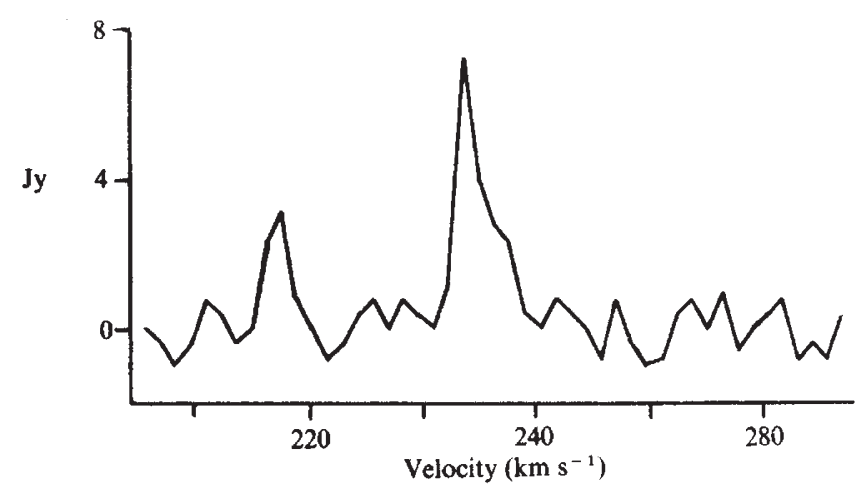

Fig. 1 Water vapour spectrum of N159 (RA 05 h $40 \mathrm{~min} 24 \mathrm{~s}$ DEC. $-69^{\circ} 46^{\prime}(0,1950.0)$. The main feature appears at $233.6 \mathrm{~km}$ $\mathrm{s}^{-1}$ with respect to the Local Standard of Rest.

Hence, one should search for water farther away from its nucleus. Hodge 47 and 52 also showed no line emission.

Our search for water masers in the direction of the Large and the Small Clouds will continue, and we hope that the results improve our knowledge not only of masers but also of our closest irregular Magellanic-type galaxies.

This research resulted from a Brazil-US bilateral scientific agreement through the national agencies CNPq and NSF, respectively. The maser receiver was developed at Haystack Observatory under the direction of Dr S. H. Zisk. Partial support was received from Brazilian science agencies FINEP and FAPESP.

Received 24 November 1980; accepted S January 1981

1. Johnston, K. J. Knowles, S. H. \& Sullivan, W. T. III Astrophys. J. 167, L93 (1971) Kaufmann, P. Zisk, S., Scalise, E. Jr, Schaal, R. E. \& Gammon, R. H. Astr. J. 82, 577 (1977)

3. Churchwell, E. et al. Astr. Astrophys. 54, 969 (1977).

4. Huchtmeier, W. K., Witzel, A., Kuhr, H., Pauliny-Toth, I. I. \& Roland, J. Astr. Astrophys. 64, L21 (1978).

5. Marques dos Santos, P. \& Lépine, J. R. D. Nature 278, 34 (1979).

6. McGee, R. X., Brooks, J. W. \& Batchelor, R. A. Aust. J. Phys. 25, 581 (1972).

7. Henize K. G. Astrophys. J. Suppl. Ser. 2, 315 (1956).

8. Hode. P. Publ astr. Soc. Pacif. 84,365 (1972).

. Hot. R astr. Soc. 173, 69P (1975).

10. Werner, M. W. et al. Mon. Not. R. astr. Soc. 184, 365 (1978).

11. Whiteoak, J. B. \& Gardiner, F. F., Mon. Not. R. astr. Soc. 174, 51 P (1976)

12. Whiteoak, J. B. \& Gardner, F. F., Mon. Not. R. astr. Soc. 176, 25P (1976)

13. Deguchi, S. Div. geol. planet. Sci. Contr. 3445 (California Institute of Technology, 1980) 14. Hyland, A. R., Thomas, J. A. \& Robinson, G. Astr. J. 83, 20 (1980). 